\title{
Newer treatments for decompensated heart failure: focus on levosimendan
}

\author{
Ferenc Follath \\ University Hospital Zürich, Steering \\ Group Drug Therapy, Zürich, \\ Switzerland
}

\begin{abstract}
Acute heart failure (AHF) is a major cause of hospitalizations. Severe dyspnea, pulmonary congestion and low cardiac output with peripheral vasoconstriction and renal hypoperfusion is a main form of clinical presentation. Most patients with acute worsening have a pre-existing decompensated chronic heart failure (ADCHF), but AHF may also occur as a first manifestation of a previously unknown heart disease. Myocardial ischemia, cardiac arrhythmias, non-compliance with medication and infections are frequent precipitating factors. Management of AHF depends on the underlying heart disease and cause of decompensation. In patients with ADCHF vasodilators and iv diuretics are first-line drugs for rapid reduction of dyspnea and congestion. In patients with signs of low cardiac output and oliguria, inotropic agents are also often administered to prevent further deterioration. Beta-adrenergic agents and phosphodiesterase inhibitors correct the hemodynamic disturbance, but may also induce arrhythmias and worsen myocardial ischemia. Inotropic therapy therefore remains controversial. A novel class of drugs, the calcium sensitizers, represent a new therapeutic option. Levosimendan was shown to improve myocardial contractility without increasing oxygen requirements and to produce peripheral and coronary vasodilation. Its therapeutic effects and tolerance have been tested in several trials. The present review focuses on the clinical pharmacology and therapeutic utility of levosimendan in patients with ADCHF.
\end{abstract}

Keywords: acute heart failure, inotropic agents, calcium sensitizers, levosimendan

\section{Introduction}

Therapeutic guidelines have been primarily developed for patients with chronic heart failure (CHF). Recommendations are based on large scale trials with angiotensin-converting enzyme (ACE) inhibitors, angiotensin receptor antagonists and beta-blockers conducted in symptomatic patients with reduced left ventricular ejection fraction, usually $<35 \%$ to $40 \%$, in a stable phase. More recently, attention also turned to acute and worsening heart failure which is a major cause of hospitalizations. Several epidemiological surveys in Europe $^{1-4}$ and in the USA ${ }^{5}$ described the demographics, symptoms, physical findings and precipitating factors of AHF. A classification according to the initial clinical presentation was proposed by the European Society of Cardiology. ${ }^{6}$ In EuroHF II $^{2}$ acute decompensation of a pre-existing $\mathrm{CHF}$ (ADCHF) with signs of pulmonary congestion and low cardiac output was the most frequent form of $\mathrm{AHF}$, occurring in $65 \%$ of patients, followed by pulmonary edema (16\%), HF and hypertension (11\%), cardiogenic shock (4\%) and right HF (3\%). In about $30 \%$ of cases AHF was the first (de novo) manifestation of a previously unknown cardiac disease. Patients with AHF require urgent therapeutic measures to reduce symptoms, improve the hemodynamic disturbancies and prevent further deterioration. Thus, drug choices, dosages and mode of administration may considerably differ from those in $\mathrm{CHF}$ where treatment can be adjusted over a longer period.

\section{Therapeutic options in ADCHF}

The initial management of AHF depends on the underlying cardiac disease, preceding drug treatment and precipitating factors. If present, hypertension, arrhythmias or acute 
coronary syndromes have to be treated first as soon as possible. Among the symptomatic measures to improve dyspnea and relieve congestion vasodilators and/or high ceiling diuretics are usually the drugs of initial choice. Nitroglycerine or isosorbide dinitrate iv have been shown to be superior to repeated high doses of furosemide in patients with pulmonary edema. ${ }^{7}$ One of the disadvantages of nitrates is a loss of efficacy due to tachyphylaxis within the first 24 to 48 hours. A new alternative for vasodilation is nesiritide, a brain natriuretic peptide (BNP) analogon, which has effects comparable to nitroglycerin without reduction of activity during prolonged administration. ${ }^{8}$ This drug has been widely used in the USA, but there were negative reports concerning a worsening renal function $^{9}$ and a trend to higher mortality under nesiritide. ${ }^{10}$

Although the increased risks have been partly refuted by a retrospective analysis ${ }^{11}$ the final value of nesiritide needs to be evaluated in further trials. If pulmonary congestion does not rapidly respond to drug treatment a noninvasive positive pressure ventilation by a face mask is a well documented effective therapeutic measure in AHF with pulmonary edema. ${ }^{12}$

One of the controversial issues in ADCHF is the use of inotropic agents. If signs of low cardiac output with peripheral vasoconstriction and oliguria persist despite correction or exclusion of a hypovolemia and patients do not respond to vasodilators and diuretics, an improvement of myocardial contractility might be needed to correct the severe cardiovascular dysfunction. For this reason beta-adrenergic drugs, dobutamine and dopamine, or phosphodiesterase inhibitors, milrinone and enoximone, are often administered. Although these agents produce a symptomatic and hemodynamic improvement, their influences on clinical outcomes are mostly negative. Increased myocardial oxygen requirements and pro-arrhythmia are well known undesired effects which may seriously compromise the utility of inotropic therapy. ${ }^{13-15}$

A new category of cardio-stimulant drugs with combined positive inotropic and vasodilating effects, the calcium sensitizers, may avoid some of the disadvantages of other inotropic agents and offer a therapeutic alternative in patients with ADCHF. Levosimendan is the best studied calcium sensitizer and is available for routine clinical use in several European countries, as well as in Brasil, Argentina and Mexico. This review will focus on the clinical pharmacology of levosimendan, its dosage, indications and analyze the results of clinical trials.

\section{Clinical pharmacology of levosimendan}

The positive inotropic action of levosimendan is due to its binding to troponin $\mathrm{C}$ which facilitates the interaction between actin and myosine filaments without changes in intracellular $\mathrm{Ca}^{++}$ion concentrations. ${ }^{16}$ In addition, the drug produces peripheral and coronary vasodilation by opening ATP-sensitive potassium channels. ${ }^{17}$ This dual mechanism of action results in an improved myocardial contractility without relevant changes in oxygen requirements ${ }^{18}$ and in a reduction of post-ischemic stunning. ${ }^{19,20}$

The pharmacokinetic and pharmacodynamic properties of levosimendan have been studied in healthy volunteers and patients with heart failure. ${ }^{21,22}$ Following intravenous administration in different dosages the pharmacokinetics remained linear with an elimination half-life of the unchanged compound of around 1 hour. Complete metabolism with formation of two pharmacologically active products (OR-1896 and OR-1855) is the mechanism of hepatic excretion. These metabolites are eliminated very slowly with half-lives of $>80$ and $>70$ hours, respectively. Because their activity is similar to that of levosimendan the pharmacodynamic effects of a 24-hour infusion last up to several days. ${ }^{23}$ For this reason more prolonged infusions are not recommended. In patients with heart failure and mild to moderate renal dysfunction, pharmacokinetics changed little, but in patients with end-stage renal disease undergoing hemodialysis, an accumulation of the active metabolites could be observed. ${ }^{24}$ Thus, initial dose reduction (starting with $0.05 \mu \mathrm{g} / \mathrm{kg} / \mathrm{min}$ ) is necessary if levosimendan is used in patients with ADCHF and renal dysfunction. There are few data available on the extent of metabolite accumulation in patients with liver disease. ${ }^{25}$ In 12 cases with alcoholic cirrhosis there was a slight prolongation of metabolite half-lives $(+30 \%)$, which would again indicate lower initial infusion rates, similar to those in patients with renal failure.

The hemodynamic effects of levosimendan have been evaluated in dose finding studies in patients with $\mathrm{CHF}^{26}$ After initial iv bolus infusions of $3,6,12,24$ or $36 \mu \mathrm{g} / \mathrm{kg}$ in 10 minutes, followed by 24 -hour continuous infusions at rates of $0.05,0.1,0.2,0.4$ and $0.6 \mu \mathrm{g} / \mathrm{kg} / \mathrm{min}$, dose-dependent increases of cardiac output and stroke volume together with a rapid decreases of pulmonary capillary wedge pressure and right atrial pressure were measured. Slight heart rate increases and reductions in mean blood pressure were also seen. Calculated peripheral vascular resistance was linearly reduced with increasing doses. Protocol defined dose limiting events were tachycardia $>20 \%$ and systolic blood pressure $<90 \mathrm{mmHg}$, mainly in patients receiving higher doses of 0.4 and $0.6 \mu \mathrm{g} / \mathrm{kg} / \mathrm{min}$. Headaches and nausea also occurred in some cases. Based on this study infusion rates 
of 0.05 to $0.2 \mu \mathrm{g} / \mathrm{kg} / \mathrm{min}$ were recommended to achieve favorable hemodynamic effects with good tolerance.

\section{Clinical trials with levosimendan}

The first randomized, double-blind, multicenter trial (LIDO) compared levosimendan with dobutamine in 203 hospitalized patients with severe low output CHF. ${ }^{27}$ Inclusion criteria were an ejection fraction $<35 \%$, a cardiac index $<2.5 \mathrm{~L} / \mathrm{min} / \mathrm{m}^{2}$ and a pulmonary capillary wedge pressure $>15 \mathrm{mmHg}$. All patients were symptomatic under a treatment with ACE inhibitors, diuretics and digoxin; one third also received a beta-blocker. Levosimendan was infused after a loading dose of $24 \mu \mathrm{g} / \mathrm{kg} / \mathrm{min}$ in 10 minutes at a rate of $0.1 \mu \mathrm{g} / \mathrm{kg} / \mathrm{min}$ for 24 hours and dobutamine without loading at $5 \mu \mathrm{g} / \mathrm{kg} / \mathrm{min}$. Dosage of both drugs was doubled after 2 hours if the intended hemodynamic improvement, defined as increase of cardiac index by $30 \%$ and reduction of pulmonary capillary wedge pressure by $25 \%$, was not achieved. This end-point was reached in $28 \%$ of patients with levosimendan compared to only $15 \%$ with dobutamine $(\mathrm{p}<0.022$ ). In the subgroup of patients under beta-blockade therapeutic targets were obtained in 10 out of 33 under levosimendan versus 3 of 29 cases under dobutamine, respectively. Furthermore, levosimendan also had a beneficial effect on clinical outcomes: at 31 days mortality was lower after levosimendan with 8 deaths (8\%) versus $17(17 \%)$ after dobutamine (hazard ratio 0.43 [0.18-1.0], p = 0.049). The prognostic benefit persisted up to 180 days, the median number of days alive and out of hospital being 157 (101-173) in the levosimendan group and 133 (43.5-169) after dobutamine $(p=0.027)$. The total number of adverse events was comparable in both treatment groups (48 vs 42 events, p 0.88), but more arrhythmias ${ }^{14}$ occurred under dobutamine than under levosimendan, ${ }^{4} \mathrm{p}<0.023$, while hypotension was observed in 9 patients under levosimendan compared to 4 under dobutamine $(\mathrm{p}=0.252)$. Thus, there was an overall advantage of levosimendan in this trial.

The second randomized clinical trial (RUSSLAN) compared levosimendan, infused for 6 hours in 4 different doses, with placebo in 504 patients with clinical and radiological signs of left ventricular failure following an acute myocardial infarction. ${ }^{28}$ The primary aim was to test the tolerability of levosimendan with the end-points hypotension and myocardial ischemia. Secondary end-points included all cause mortality and worsening HF. At doses of 0.1 to $0.2 \mu \mathrm{g} / \mathrm{kg} / \mathrm{min}$ levosimendan did not increase the incidence of hypotension or ischemia and reduced the combined risk of death and worsening HF during the first 24 hours (4\% versus
$8.8 \%$ in the placebo group, $\mathrm{p}=0.044)$. There was, however, a non-significant trend for a higher risk of hypotension and/or ischemia at the highest dose $(0.4 \mu \mathrm{g} / \mathrm{kg} / \mathrm{min})$ of levosimendan. Mortality at 14 days was $19.6 \%$ after placebo compared to $10.0 \%$ to $13.1 \%$ (average $11.7 \%$ ) in the four subgroups receiving levosimendan $(\mathrm{p}=0.031)$, but after 180 days the differences were no more statistically significant.

More recently, two larger randomized, double-blind trials were conducted in the USA and in Europe. In REVIVE II, 24-hour infusions of levosimendan were compared with placebo in 600 patients with ADCHF. ${ }^{29}$ The primary 'composite' end-point was a measure of the clinical course, based on subjective and objective symptom assessments over 5 days. Levosimendan was given after an initial loading of $12 \mu \mathrm{g} / \mathrm{kg}$ in 10 minutes followed by a uniform infusion rate of $0.2 \mu \mathrm{g} / \mathrm{kg} / \mathrm{min}$ for 24 hours. Co-administration of iv diuretics and vasodilators was allowed without hemodynamic monitoring. At 5 days 33\% more patients improved and 26\% fewer worsened after levosimendan than placebo $(p=0.015)$. This positive clinical outcome was, however, partially neutralized by a high incidence of hypotension ( 50.2 vs $36.4 \%$, $\mathrm{p}<0.01)$ and more frequent atrial fibrillation (8.5 vs $2.0 \%)$ under levosimendan and placebo, respectively. There was also a non-significant negative trend for higher mortality at 14 and 31 days following levosimendan. The SURVIVE trial ${ }^{30}$ compared levosimendan with dobutamine in 1327 patients with acute heart failure judged to require inotropic support. Again, levosimendan was given in a uniform high dosage at $0.2 \mu \mathrm{g} / \mathrm{kg} / \mathrm{min}$ to all patients for 24 hours; the average dose of dobutamine was $6 \mu \mathrm{g} / \mathrm{kg} / \mathrm{min}$. Patients were hospitalized at intensive care units but not invasively monitored. Also in this trial iv diuretics and concomitant vasodilators were allowed. The primary end-point was survival at 180 days. At the end of follow up there were 173 deaths $(26 \%)$ in the levosimendan group as compared to $185(28 \%)$ on placebo $(\mathrm{p}=0.4)$. However, a subgroup analysis at 31 days showed a survival advantage of levosimendan in patients with a prior history of heart failure. Also plasma BNP levels decreased significantly more at 24 hours and at 3 to 5 days after levosimendan than after dobutamine. The rates of serious adverse events were not different in the two treatments groups, but there were more hypotensions and higher heart rate increases under levosimendan, while patients were less likely to experience worsening cardiac failure than under dobutamine.

Following the presentation of REVIVE II and SURVIVE doubts were voiced regarding the utility of levosimendan in the treatment of AHF. ${ }^{31}$ For this reason it is important to analyze the basic differences between the initially positive 
and the more recent partly contradictory trial results and try to explain the variable outcomes. In the LIDO trial all patients were hemodynamically monitored and had initial capillary wedge pressures of $15 \mathrm{mmHg}$ or more (mean $25 \pm 8 \mathrm{mmHg}$ ), as well as a cardiac index $<2.5 \mathrm{~L} / \mathrm{kg} / \mathrm{m}^{2}$. Thus, they all belonged to a well defined category of ADCHF patients; cases with signs of active myocardial ischemia or acute arrhythmias were excluded. Co-administration of iv diuretics or vasodilators was not allowed. In contrast, both in REVIVE and SURVIVE, simultaneous treatment with the above drugs, often at high doses, was permitted without invasive monitoring. Hence, a drug-induced hypovolemia could not be excluded and was probably present in many cases. The high incidence of hypotension (36.4\%) even under placebo in REVIVE II would fit this assumption. Administering levosimendan, with its marked vasodilating effect at high and uniform doses, especially to heavily pretreated patients, can be expected to result in marked drops in blood pressure and also lead to cardiac arrhythmias in patients with an underlying coronary artery disease which in SURVIVE was present in over 60\%. Hypokalemia due to intensive diuretic administration was an other potentially negative factor. Thus, in retrospect the design in both trials can be questioned. The main lesson from these two recent trials is that a high initial infusion rate of levosimendan has to be avoided and pre-existing hypovolemia be excluded before starting this treatment. In critical cases, especially with systolic blood pressure values $<100 \mathrm{mmHg}$, infusion should be initiated without loading at only $0.05 \mu \mathrm{g} / \mathrm{kg} / \mathrm{min}$ under close observation to avoid severe hypotension.

\section{Levosimendan in routine clinical practice}

There are also observational studies available documenting the practical experience with levosimendan in everyday practice. A Brazilian cohort ${ }^{32}$ including 182 high risk patients with decompensated HF received open label levosimendan infusions added to a baseline treatment with diuretics, ACE inhibitors/angiotensin receptor antagonists and beta-blockers. In $39 \%$ inotropic agents were already used before without success. Out of the 182 patients 139 (76.4\%) responded, defined by reduction of dyspnea and of auscultatory signs of pulmonary congestion and could be discharged from hospital. Treatment success was even better $(83.3 \%)$ in patients on beta-blockers $(21 \%)$. Levosimendan was said to be well tolerated; no severe hypotension or an increase in cardiac arrhythmias occurred. Nevertheless, 54 patients had some adverse events which resolved spontaneously or after dose reduction. Mortality rate during hospitalization was $14.8 \%$ in patients responding to levosimendan compared to $62.8 \%$ in non-responders. A similar positive practical experience was reported from Portugal. ${ }^{33}$ In an open, prospective, multicenter study in 129 hospitalized patients with systolic HF and signs of decompensation, a 24-hour infusion of levosimendan at a dose of 0.05 to $0.1 \mu \mathrm{g} / \mathrm{kg} / \mathrm{min}$ was given as a first line inotropic therapy. During an observation period of 5 days a well defined clinical improvement (reduction of NYHA class and increased diuresis with reduction of serum creatinine) without additional inotropes and no adverse events was obtained in $80 \%$. These observational data indicate that levosimendan can be safely and successfully used under everyday routine clinical conditions.

\section{Levosimendan and renal function}

An important advantage of levosimendan is its potential to improve renal function in patients with ADCHF. A significant reduction of serum creatinine was reported already in the initial clinical trial. ${ }^{27}$ Subsequently, two studies were specially conducted to evaluate the effects of levosimendan on serum creatinine and glomerular filtration rate (GFR). In 40 patients with advanced $\mathrm{CHF}$ and increased serum creatine values $(1.92 \pm 0.13 \mathrm{mg} / \mathrm{dL})$, a 24 -hour infusion at a dose of $0.1 \pm \mathrm{g} / \mathrm{kg} / \mathrm{min}$ compared to a control group without inotropic support, but otherwise identical treatment, showed a reduction of serum creatinine by $-0.32 \pm 0.28 \mathrm{mg} / \mathrm{dL}$ and $-0.01 \pm 0.13 \mathrm{mg} / \mathrm{dL}$, respectively. ${ }^{34}$ After 3 months creatinine clearance was still improved from the initial value of $43.7 \pm 2.9 \mathrm{~mL} / \mathrm{min}$ to $53.6 \pm 8.6 \mathrm{~mL} / \mathrm{min}$, whereas in the control group GFR did not change. In another study levosimendan was compared with dobutamine, both infused for 24 hours, in 88 consecutive patients with ADCHF. ${ }^{35}$ Within 24 hours GFR increased by $15.3 \%$ under levosimendan versus $-1.33 \%$ under dobutamine. GFR values improved further after 72 hours.

\section{Effects of levosimendan on surrogate end-points in HF}

Levosimendan was also shown in smaller comparative trials to have potentially beneficial effects not only on neurohormones, but also on inflammatory cytokines and apoptosis mediators in HF. Moertl et $\mathrm{al}^{36}$ observed a BNP reduction by $28 \%$ at 24 hours and by $22 \%$ at 48 hours following 24 hours levosimendan infusion in patients with decompensated $\mathrm{HF}$, but these effects disappeared after 1 week. Parissis et $\mathrm{al}^{37}$ reported a significant $\mathrm{BNP}$ reduction together with further reduction of interleukin-6 (IL-6). In their patients 
there was also an improvement of left ventricular diastolic function as judged by echocardiographic techniques. Trikas et $\mathrm{al}^{38}$ equally found significant decreases of serum IL-6, TNF-alfa as well as of the apoptosis marker soluble FAS (sFAS). Such surrogate effects could have positive long-term therapeutic influences for levosimendan in addition to its cardio-stimulatory activity, but convincing clinical data in this respect are still lacking.

\section{Emerging indications and treatments}

There are also some new indications for levosimendan treatment, such as repetitive administration in patients with advanced $\mathrm{HF},{ }^{39,40}$ post-operative myocardial dysfunction following cardiac surgery, ${ }^{41}$ or right ventricular failure. ${ }^{42}$ These interesting applications require further larger trials to confirm the therapeutic utility of levosimendan.

The recently developed new inotropic and lusitropic agent, istaroxime, which has similar hemodynamic effects and also does not seem to increase myocardial oxygen requirements, ${ }^{43}$ might become a potential alternative to levosimendan in patients with HF, but the clinical data are still limited.

\section{Conclusions}

\section{Current indications and dosage recommendations for levosimendan}

Levosimendan represents a new and valuable option for the treatment of properly selected and monitored patients with decompensated heart failure needing inotropic support to prevent further deterioration. The best documented indication for levosimendan is the treatment of patients with ADCHF presenting with high filling pressures, signs of congestion and reduced cardiac output with peripheral vasoconstriction. The combined inodilating effects of levosimendan rapidly relieve pulmonary congestion and improve organ perfusion, including renal blood flow and GFR. However, before starting a levosimendan infusion it is essential to exclude and correct a hypovolemia due to preceding high dose iv diuretics or vasodilators, otherwise an arterial hypotension may occur. Low serum potassium should also be corrected to avoid proarrhythmic effects.

Based on clinical experience in countries in which use is widespread, a loading dose of levosimendan can be omitted in many patients, especially if the initial systolic blood pressure is $\leq 100 \mathrm{mmHg}$. In view of the short ( 1 hour) halflife of unchanged levosimendan a near maximum effect of a given infusion rate will be reached within 4 hours without loading. A starting infusion rate of $0.05 \mu \mathrm{g} / \mathrm{kg} / \mathrm{min}$ is usually safe even in critical patients. The dose can be increased to $0.1 \mu \mathrm{g} / \mathrm{kg} / \mathrm{min}$ after 1 to 2 hours if the drug is well tolerated; $0.2 \mu \mathrm{g} / \mathrm{kg} / \mathrm{min}$ might be needed in some patients not adequately responding. The standard duration of infusion is 24 hours, but in some situations, such as intermittent ambulatory administration, a 6- to 8-hour infusion may be more suitable. A prolonged infusion is not recommended, since the slowly formed active metabolites maintain the hemodynamic effects for several days and longer treatments lead to drug accumulation with tachycardia and hypotension. ${ }^{42}$

The best efficacy can be expected in patients pre-treated with beta-blockers which, in contrast to beta-adrenergic drugs, do not reduce the actions of levosimendan. In patients not yet under beta-blockers these drugs can be safely introduced and uptitrated under protection with levosimendan even after an episode of acute decompensation.

\section{Disclosures}

The author has received lecture fees from, and consulted for, Abbott Laboratories.

\section{References}

1. Rudiger A, Harjola VP, Müller A, et al. Acute heart failure: clinical presentation, one-year mortality and prognostic factors. Eur J Heart Fail. 2004;7:662-670.

2. Nieminen MS, Brutsaert D, Dickstein K, et al. EuroHeart Failure Survey II: a survey on hospitalised acute heart failure patients: description of population. Eur Heart J. 2006;27:2725-2736.

3. Tavazzi L, Maggioni A, Lucci D, et al. Nationwide survey on acute heart failure in cardiology ward services in Italy. Eur Heart J. 2006;27:1207-1215.

4. Siirelä-Waris K, Lassus J, Melin J, Peuhkurinen K, Nieminen MS, Harjola VP. Characteristics, outcomes, and predictors of 1-year mortality in patients hospitalised for acute heart failure. Eur Heart J. 2006;27:3011-3017.

5. Adams KF, Fonarow GC, Emermann CL, et al. Characteristics and outcome of patients hospitalised for heart failure in the United States: rationale, design and preliminary observations from the first 100,000 cases in the Acute Decompensated Heart Failure National Registry (ADHERE). Am Heart J. 2005;149:209-216.

6. The Task Force for the Diagnosis and Treatment of Acute and Chronic Heart Failure of the European Society of Cardiology. ESC guidelines for the diagnosis and treatment of acute and chronic heart failure 2008. Eur Heart J. 2008;29:2388-2442.

7. Cotter G, Metzkor E, Kaluski E, et al. Randomised trial of high-dose isosorbide dinitrate plus low-dose furosemide versus high-dose furosemide plus low-dose isosorbide dinitrate in severe pulmonary oedema. Lancet. 1998;351:389-393.

8. The VMAC investigators. Intravenous nesiritde vs nitroglycerine for the treatment of decompensated heart failure. JAMA. 2002;287:1531-1540.

9. Sackner-Bernstein JD, Skopicki HA, Aaronson KD. Risk of worsening renal function with nesiritide in patients with acutely decompensated heart failure. Circulation. 2005:111:1487-1491.

10. Sackner-Bernstein JD, Kowalski M, Fox M, Aaronson K. Short term risk of death after treatment with nesiritide for decompensated heart failure. A pooled analysis of randomised controlled trials. JAMA. 2005;293:1900-1905. 
11. Arora S, Clarke K, Srinivasan V, Gradman A. Effect of nesiritide on renal function in patients admitted for decompensated heart failure. QJM. 2007;100:699-706.

12. Peter JV, Moran JL, Philips-Hughes J, Graham P, Bersten AD. Effect of non-invasive positive pressure ventilation (NIPPV) on mortality in patients with acute cardiogenic pulmonary oedema: a meta-analysis. Lancet. 2006;367:1155-1162.

13. O'Connor CM, Gattis WS, Uretsky BF, et al. Continuous intravenous dobutatmine is associated with an increased risk of death in patients with advanced heart failure: Insights from the Flolan International Randomised Survival Trial ( FIRST). Am Heart J. 1999;138:78-86.

14. Thackray S, Easthaugh J, Fremantle N, et al. The effectiveness and relative effectiveness of intravenous inotropic drugs acting through the adrenergic pathway in patients with heart failure - meta-regression analysis. Eur J Heart Fail. 2002;4:515-529.

15. Cuffe MS, Califf MR, Adams KF, et al. Short-term intravenous milrinone for acute exacerbation of chronic heart failure. JAMA. 2002;287:1541-1547.

16. Haikala H, Kaivola J, Nissinen E, et al. Cardiac troponin C as a target protein for a novel calcium sensitizing drug, levosimendan. J Mol Cell Cardiol. 1995;27:1859-1866.

17. Yokoshiki H, Kutsube Y, Sunagawa M, et al. The novel calcium sensitizer levosimendan activates the ATP-sensitive $\mathrm{K}+$ channel in rat ventricular cells. J Pharmacol Exp Ther. 1997;283:375-383

18. Ukkonen H, Saraste M, Akkila J. Myocardial efficiency during levosimendan infusion in congestive heart failure. Clin Pharmacol Ther. 2000;68:522-531.

19. Jamali IN, Kersten JR, Pagel PS, et al. Intracoronary levosimendan enhances contractile function of stunned myocardium. Anaesth Analg. 1997;85:23-29

20. Sonntag S, Sudberg S, Lehtonen LH, Kleber FX. The calcium sensitizer levosimendan improves the function of stunned myocardium after percutaneous transluminal coronary angioplasty in acute myocardial ischemia. J Am Coll Cardiol. 2004;43:2177-2182.

21. Lilleberg J, Sundberg S, Häyhä M, et al. Haemodynamic dose-efficacy of levosimendan in healthy volunteers. Eur J Clin Pharmacol. 1994;47:267-274.

22. Kivikko M, Antila S, Eha J, Lehtonen L, Pentikäinen PJ. Pharmacodynamics and safety of a new calcium sensitzer, levosimendan, and its metabolites during an extended infusion in patients with severe heart failure. J Clin Pharmacol. 2002;42:43-51.

23. Lilleberg J, Laine M, Palkama T, Kivikko M, Pohjanjousi P, Kupari M. Duration of the haemodynamic action of a 24-h infusion of levosimendan in patients with congestive heart failure. Eur J Heart Fail. 2007;9:75-82.

24. Puttonen J, Kantele S, Kivikko M, et al. Effect of severe renal failure and haemodialysis on the pharmacokinetics of levosimendan and its metabolites. Clin Pharmacokinet. 2007;46:235-246.

25. Puttonen J, Kantele S, Ruck A, et al. Pharmacokinetics of intravenous levosimendan and its metabolites in subjects with hepatic impairment. J Clin Pharmacol. 2008;48:445-454.

26. Nieminen MS, Akkila J, Hasenfuss G, et al. Haemodynamic and neurohumoral effects of continuous infusion of levosimendan in patients with congestive heart failure. J Am Coll Cardiol. 2000;36:1903-1912.

27. Follath F, Cleland JGF, Just H, et al. Efficacy and safety of intravenous levosimendan compared with dobutamine in severe low-output heart failure (the LIDO study): a randomised double-blind trial. Lancet. 2002;360:196-202.
28. Moiseyev VS, Poder P, Andrejevs N, et al. Safety and efficacy of a novel calcium sensitizer, levosimendan, in patients with left ventricular failure due to an acute myocardial infarction. A randomised, placebo controlled, double blind study (RUSSLAN). Eur Heart J. 2002;23:1422-1432.

29. Packer M. REVIVE II: Multicenter placebo controlled trial of levosimendan on clinical status in acutely decompensated heart failure. American Heart Association Scientific Sessions 2005, November 13-16, Dallas, Texas. Late breaking clinical trials II. Circulation. 2005; $112: 3363$.

30. Mebazaa A, Nieminen MS, Packer M, et al. Levosimndan vs dobutamine for patients with acute decompensated heart failure. The SURVIVE randomised trial. JAMA. 2007;297:1883-1891.

31. Petersen JW, Felker GM. Inotropes in the management of acute heart failure. Crit Care Med. 2008;36(1 Suppl):S106-S111.

32. Bocchi AE, Vilas-Boas F, Moreira MC, et al. Levosimendan in decompensated heart failure patients: efficacy in a Brasilian Cohort. Results of the BELIEF study. Arq Bras Cardiol. 2008;90:182-190.

33. Silva-Cardoso J, Ferriera J, Prazeredes de Sà E, et al. Levosimendan in daily intensive care practice- the experience of 15 centres. Background, methods and organisation of the PORTLAND study. Rev Port Cardiol. 2004;23:1431-1443.

34. Zemljic G, Bunc M, Yazdanbakhsh AP, Vrtovec B. Levosimendan improves renal function in patients with advanced heart failure awaiting cardiac transplantation. J Card Fail. 2007;13:417-421.

35. Yilmaz MB, Yalta K, Yontar C, et al. Levosimendan improves renal function in patients with acute decompensated heart failure: comparison with dobutamine. Cardiovasc Drugs Ther. 2007;21:231-435.

36. Moertl D, Berger R, Huelsmann B, et al. Short-term effects of levosimendan and prostaglandin E1 on hemodynamic parameters and B-type natriuretic peptide levels in patients with decompensated chronic heart failure. Eur J Heart Fail. 2005;7:1156-1163.

37. Parissis JT, Panou F, Farmakis D, et al. Effects of levosimendan on markers of left ventricular diastolic function and neurohumoral activation in patients with advanced heart failure. Am J Cardiol. 2005;96:423-426.

38. Trikas A, Antoniades C, Latsios G, et al. Log-term effects of levosimendan infusion on inflammatory processes and sFAS in patients with severe heart failure. Eur J Heart Fail. 2006;8:804-809.

39. Berger R, Moertl D, Huelsmann M, et al. Levosimendan and prostaglandin E1 for uptitration of betablockade in patients with advanced chronic heart failure. Eur J Heart Fail. 2007;9:202-208.

40. Nanas JN, Papazouglu PP, Tsagalou EP, et al. Efficacy and safety of intermittent, long-term, concomitant dobutamine and levosimendan infusions in severe heart failure refractory to dobutamine alone. $\mathrm{Am} \mathrm{J}$ Cardiol. 2005;95:768-771.

41. De Hert SG, Losomradee S, Cromheecke S, Van der Linden PJ. The effects of levosimendan in cardiac surgery patiens with poor left ventricular function. Anaesth Analg. 2007;104:766-773.

42. Poelzl G, Zwick RH, Grander W, et al. Safety and effectiveness of levosimndan in patients with predominant right heart failure. Herz. 2008;33:368-373.

43. Georghiade M, Blair JEA, Filippatos GS, et al. Hemodynamic, echocardiographic, and neurohumoral effects of istaroxime, a novel intravenous inotropic and lusitropic agent. J Am Coll Cardiol. 2008;51:2276-2285. 